\title{
Atorvastatin Sodium
}

National Cancer Institute

\section{Source}

National Cancer Institute. Atorvastatin Sodium. NCI Thesaurus. Code C78676.

The sodium salt of atorvastatin, a synthetic lipid-lowering agent. Atorvastatin competitively inhibits hepatic hydroxymethyl-glutaryl coenzyme A (HMG-CoA) reductase, the enzyme which catalyzes the conversion of HMG-CoA to mevalonate, a key step in cholesterol synthesis. This agent increases the number of LDL receptors on hepatic cell surfaces, enhancing the uptake and catabolism of LDL and reducing LDL production and the number of LDL particles, and lowers plasma cholesterol and lipoprotein levels. Like other statins, atorvastatin may also display direct antineoplastic activity, possibly by inhibiting farnesylation and geranylgeranylation of proteins such as small GT P-binding proteins, which may result in the arrest of cells in the G1 phase of the cell cycle. This agent may also sensitize tumor cells to cyctostatic drugs, possibly through the mTORdependent inhibition of Akt phosphorylation. 